\title{
Congenital Absence of the Left Circumflex Artery With Super-Dominant Right Coronary Artery: Extremely Rare Coronary Anomaly
}

\author{
Shakil Sattar Ahmed Shaikha, b, Vikrant Deshmukha, Vishal Patila, Zahidullah Khan ${ }^{\mathrm{a}}$, \\ Rahul Singla ${ }^{\text {a }}$, Narender Omprakash Bansal ${ }^{\mathrm{a}}$
}

\begin{abstract}
Among the congenital anomalies of the coronary arteries, absent left circumflex artery (LCX) defect is extremely rare. Only a few cases have been reported in the literature. We report a case of a 48-yearold female who presented with a 4-month history of exertional chest pain with positive stress (treadmill) test. Conventional coronary angiogram showed a normal left anterior descending, absent LCX and a super-dominant right coronary artery (RCA) with prominent branches. Aortography also failed to show a separate ostium for the left circumflex artery. Multi-detector computed tomographic coronary angiography was performed to confirm the diagnosis of congenital absence of the LCX. It is a benign incidental finding, however some patients present with angina-like symptoms often resulting in detection of this rare anatomy on coronary angiography. Precise morphological and functional evaluation of the anomalous coronary artery is important for selecting the best treatment modality and better prognosis.
\end{abstract}

Keywords: Chest pain; Left circumflex artery; Coronary angiography; Coronary vessel anomalies; Congenital abnormalities

\section{Introduction}

Congenital coronary artery anomalies, infrequently seen during coronary angiographic studies, are mostly diagnosed incidentally. The prevalence in different studies ranges from $0.6 \%$ to $1.3 \%$ [1]. Congenital absence of the left circumflex coronary artery (LCX) is an extremely rare coronary artery anomaly in which the artery fails to develop in the left atri-

\footnotetext{
Manuscript submitted June 20, 2018, accepted June 27, 2018

aDepartment of Cardiology, Grant Medical College, Sir JJ group of Hospital, Mumbai, India

${ }^{b}$ Corresponding Author: Shakil Sattar Ahmed Shaikh, 201, Ayesha Manzil, A Wing Sunrise Galaxy Housing Society, Dr.Ambedkar Road, Kalyan (West), Maharashtra, India. Email: drshakilsshaikh@rediffmail.com
}

doi: https://doi.org/10.14740/cr749w oventricular groove. Yamanaka and Hobbs found it in only 4 of 126,595 patients who underwent coronary angiography and with a frequency of only $0.003 \%$ in all the patients [1]. Computed tomography (CT) coronary angiography is advantageous over catheter angiography in differentiating between congenital absence and complete obstruction. It also helps in delineating the course of the vessel in relation to the surrounding cardiac chambers [2]. Here we present a case of a 48-year-old female with exertional chest pain and coronary angiography revealed absent LCX with super-dominant right coronary artery (RCA), which was later confirmed on CT coronary angiography.

\section{Case Report}

A 48-year-old lady, non-hypertensive and non-diabetic, presented with chest pain on exertion since 4 months. Physical examination did not reveal any abnormal findings. Cardiovascular examination was normal. Laboratory parameters for lipid and renal function were normal. Her electrocardiogram showed a sinus rhythm with no significant ST-T changes. Echocardiography revealed no regional wall motion abnormalities with left ventricular ejection fraction of $60 \%$. Stress (treadmill) test showed positive result for inducible ischemia by Bruce protocol at 9 METS. So she was further investigated for coronary artery disease. Coronary angiography revealed that the left main and left anterior descending (LAD) coronary arteries appeared normal (Fig. 1, 2). The RCA was a large super-dominant vessel which crossed the left atrioventricular groove and supplied the inferior and posterolateral walls of the left ventricle (Fig. 3, 4). The LCX was not visualised (Fig.1, 2). Aortic root angiography did not reveal any anomalous LCX (Fig. 5). However, angiography using a 64-slice multi-detector computed tomography (MDCT) performed on the following day confirmed absence of the LCX. The posterior descending artery (PDA) and posterior left ventricle (PLV) branches were prominent. The PLV supplied the posterolateral wall of the left ventricle (Fig. 6). Hence patient was diagnosed a case of congenital absence of the LCX with superdominant RCA without evidence of atherosclerotic coronary artery disease. The patient was advised medical management and regular follow-up. 


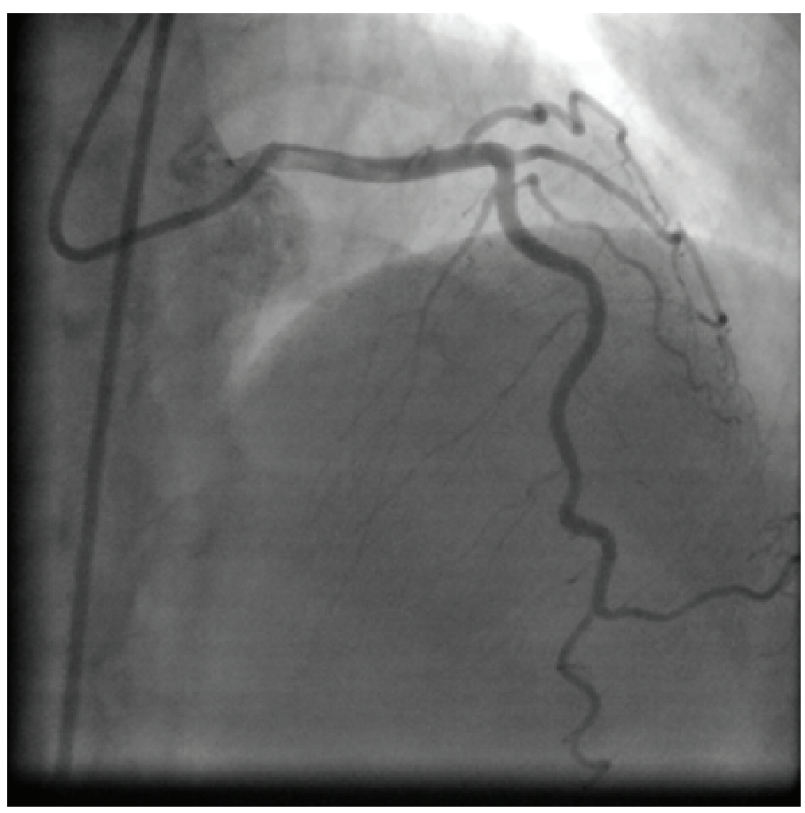

Figure 1. Left coronary angiogram in right anterior oblique (RAO) cranial view: no circumflex artery is demonstrated.

\section{Discussion}

In the literature, the absence of LCX is usually regarded as an extremely rare anomaly of coronary arteries. The congenital absence of the LCX is results from the failure of LCX development in the left atrioventricular groove. However, some authors believe that this condition is not a real congenital absent anomaly so it is defined as anomalous origin of the LCX from distal RCA $[3,4]$. Almost always this anomaly is defined as a benign

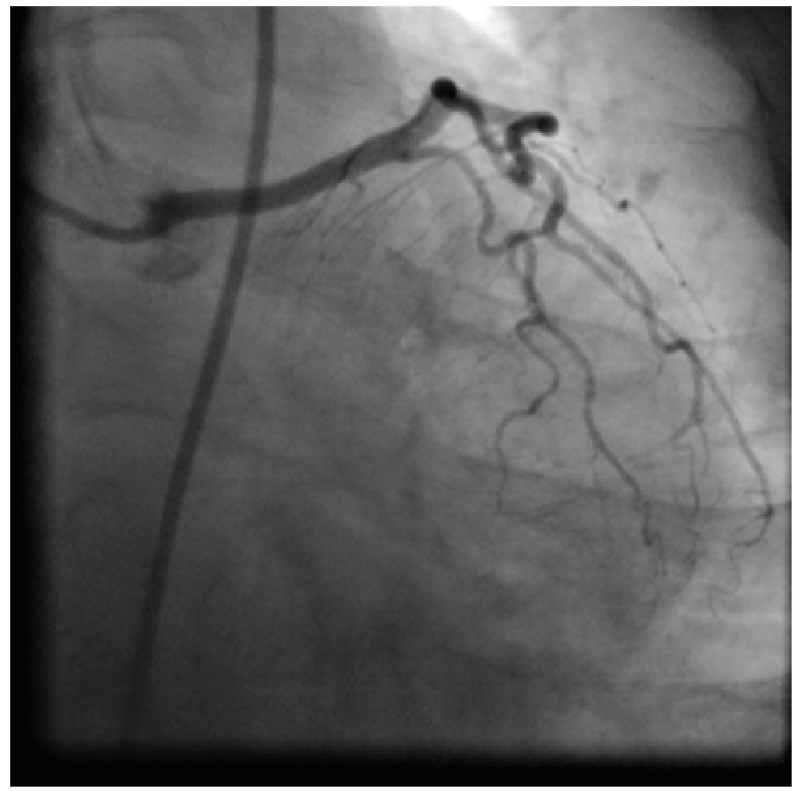

Figure 2. Left coronary angiogram in RAO caudal view: showing absent left circumflex (LCX) and long left main coronary artery.

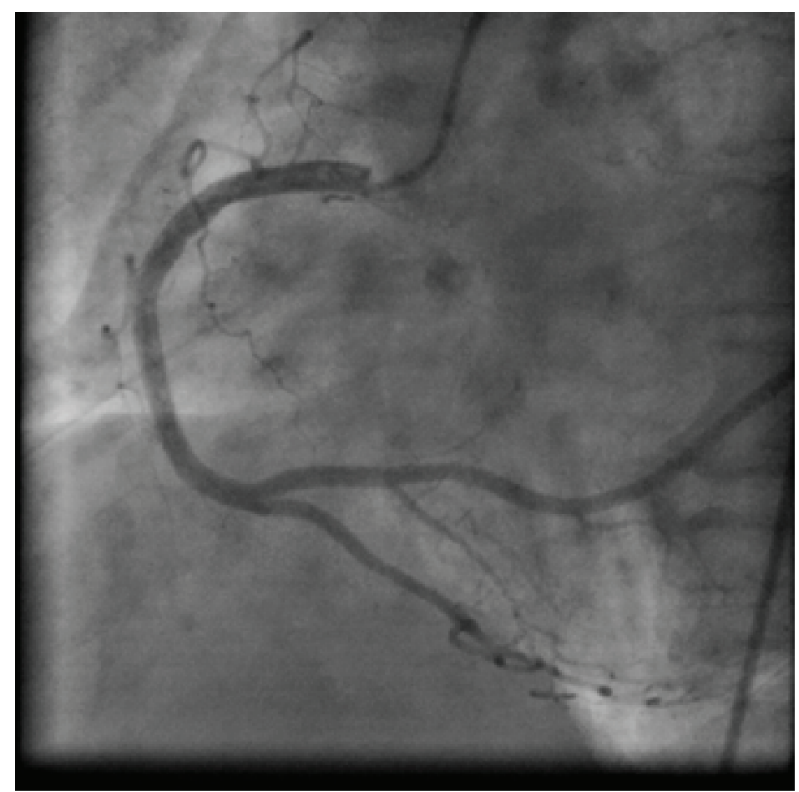

Figure 3. Right coronary angiogram in left anterior oblique (LAO) view: showing super-dominant RCA with large posterolateral branches supplying the area of left circumflex territory.

condition and has no significant clinical symptom. However, it can cause angina-like symptoms, particularly on exertion. Most of reported cases of this condition are claimed to have experienced chest pain on exertion; our case did too. The etiology of this angina-like symptom is not exactly known. One hypothesis that is accepted as an explanation of the pathophysiology of congenitally absent LCX is steal phenomenon [5-7]. This

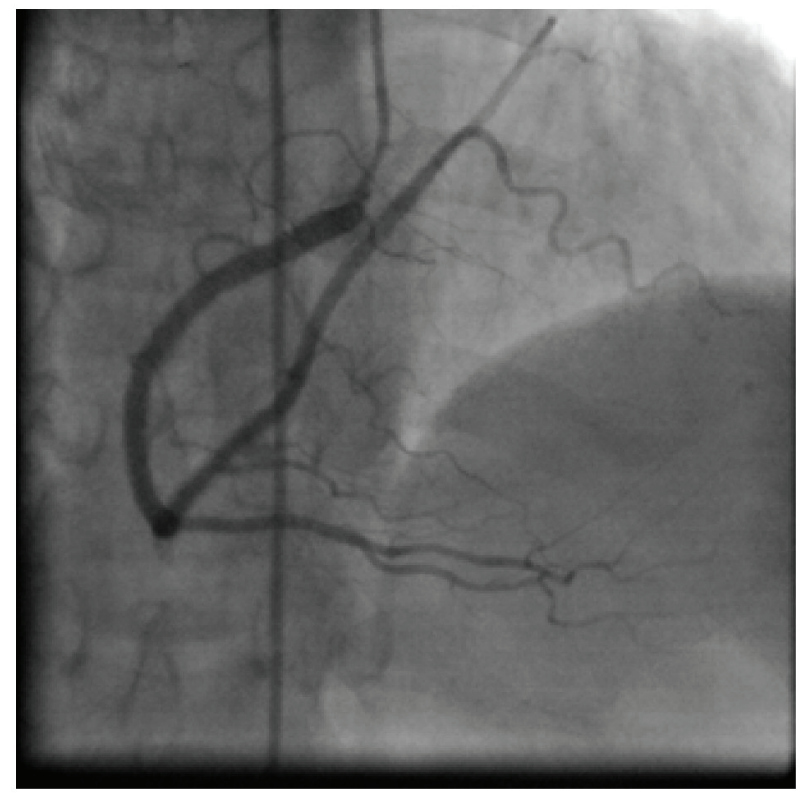

Figure 4. Right coronary angiogram in RAO cranial view: showing a super-dominant RCA with large posterolateral branches perfusing the posterolateral and lateral walls of the heart (usually supplied by the left circumflex artery). 


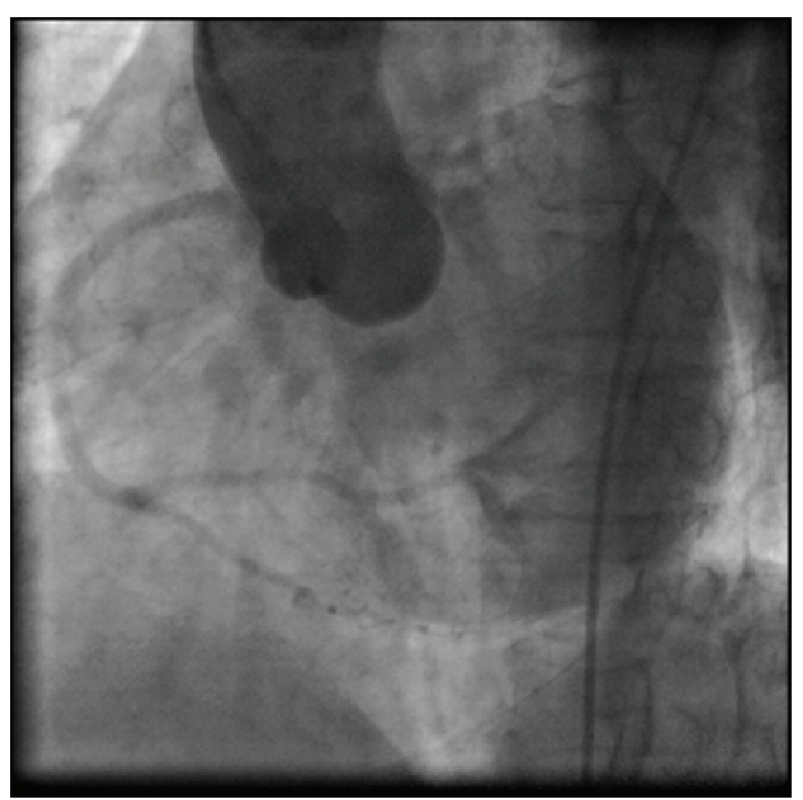

Figure 5. Aortic root angiogram in LAO caudal view: showing no evidence of the anomalous origin of left circumflex artery.

phenomenon causes an increased arterial supply to the LCX territory, and results in transient ischemia of other coronary arterial territories, resulting in perfusion defects in the stress thallium-201 myocardial perfusion study [5]. However, the true cause of the angina-like symptoms in patients with absent LCX is hard to prove and only can be suggested by circumstantial evidence. When the LCX is absent, RCA dominance is important. Normally, the LCX and RCA run around the atrioventricular groove and form a circle. If a patient has a small LCX, a well-developed RCA ascends the posterior atrioventricular groove beyond the crux and perfuses the left ventricular free wall. In fact, the present patient had a very well-developed, super-dominant RCA and in cases with a dominant compensating artery, the absence of a coronary artery is not of clinical significance as long as coronary artery disease does not develop. It has been reported that congenital absence of the LCX might be associated with systolic click syndrome and could present with chest pain, episodic rapid heartbeats, and syncope [5]. One case report describes a patient with absent LCX who presented with myocardial ischemia due to $90 \%$ occlusion of the superdominant RCA, resulting in inferior, posterior and lateral wall myocardial infarction, which they reported as being equivalent to two-vessel disease involving both the RCA and LCX [8]. Other coronary anomalies have been associated with significant cardiac symptoms i.e. coronary anomalies where the left coronary artery originates from the right coronary sinus or the right coronary artery originates from the left coronary sinus have been associated with sudden cardiac death during exertion; this occurs because exercise causes the aortic root and pulmonary trunk to dilate, resulting in compression of the anomalous vessel, which courses between the aorta and pulmonary trunk [9]. Anomalous origin of coronary arteries, particularly the LCX, have been associated with accelerated atherosclerosis, believed to be due to increased mechanical trauma, abnormal wall stress,

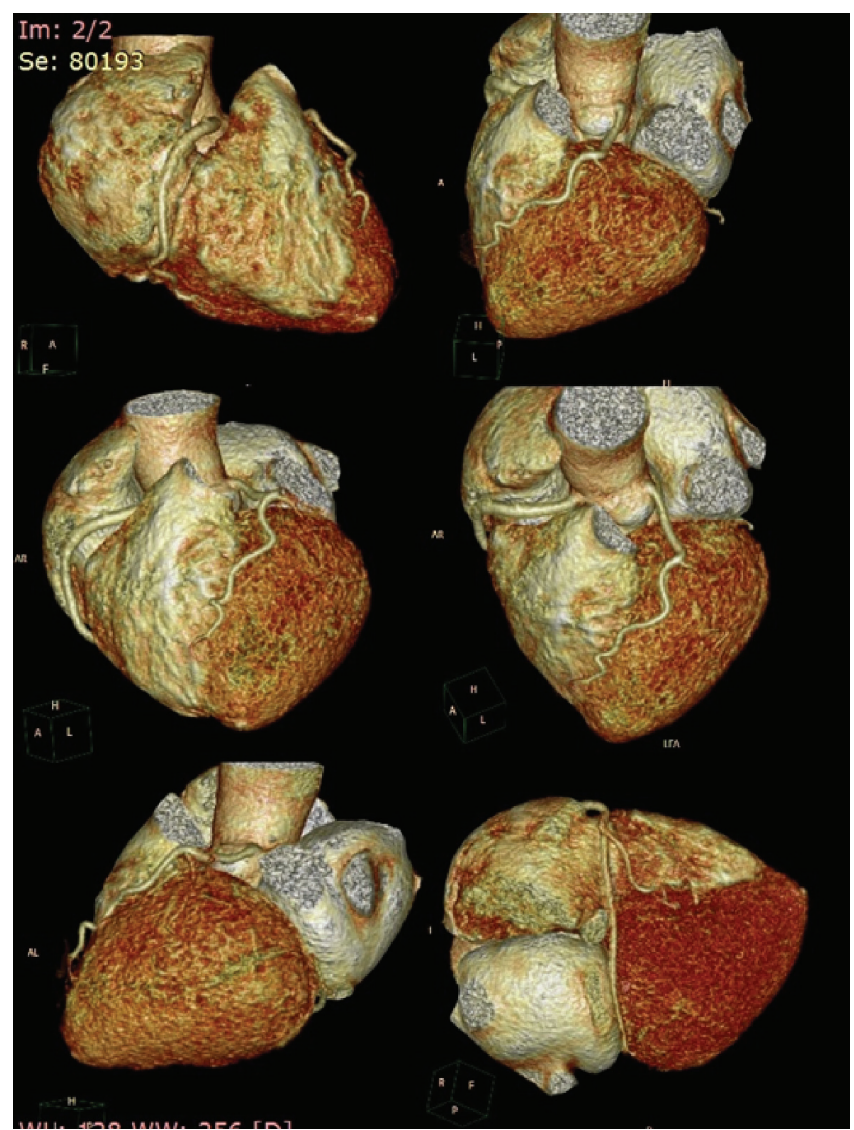

Figure 6. Different views of three-dimensional computed tomography scan shows absent left circumflex artery in the atrioventricular groove with single left anterior descending artery with super-dominant right coronary artery with a good-sized right posterolateral ventricular branch crossing the crux of the heart and then ascending into the inferior part of atrioventricular groove and supplying the posterolateral and lateral walls of the heart (usually supplied by the left circumflex artery).

and abnormal flow strain on the anomalous vessels [10]. While the absence of a LCX has yet to be significantly associated with any major cardiac event, identification of patients with this anomaly is crucial when performing cardiac interventions because patients with an absent LCX (or other coronary anomalies) are at increased risk of being misdiagnosed during cardiac catheterization procedures (e.g. congenitally absent LCX mistaken for $100 \%$ occlusion of the LCX), and they require extra care when performing cardiac bypass procedures to avoid accidental ligation or transection of anomalous vessels and to ensure that the grafts are placed properly to restore perfusion to ischemic myocardium $[11,12]$.

\section{Conclusions}

Recognition of this anomaly is important, as it may mimic atherosclerotic coronary artery disease, where oxygen demand in myocardium normally supplied by it is taken over by RCA and such supply may not be sufficient during increased physical activity. Additionally, atherosclerotic lesions may be more im- 
portant in such patients because of diminished compensating mechanisms.

\section{Acknowledgments}

We are grateful to Dr. Narender Omprakash Bansal, professor and head of the Department of Cardiology, Sir JJ Group of Hospital, Mumbai, India, for allowing us to publish this case report.

\section{Guarantor Statement}

Dr Shakil Sattar Ahmed Shaikh will act as the guarantor of the manuscript.

\section{Conflict of Interest}

None.

\section{Funding Support}

None.

\section{References}

1. Yamanaka O, Hobbs RE. Coronary artery anomalies in 126,595 patients undergoing coronary arteriography. Cathet Cardiovasc Diagn. 1990;21(1):28-40.

2. Hongsakul K, Suwannanon R. Congenital absence of left circumflex artery detected by computed tomography coronary angiography: a case report. Case Rep Vasc Med.
2012;2012:204657.

3. Erol C, Seker M. Coronary artery anomalies: the prevalence of origination, course, and termination anomalies of coronary arteries detected by 64-detector computed tomography coronary angiography. J Comput Assist Tomogr. 2011;35(5):618-624.

4. Shriki JE, Shinbane JS, Rashid MA, Hindoyan A, Withey JG, DeFrance A, Cunningham M, et al. Identifying, characterizing, and classifying congenital anomalies of the coronary arteries. Radiographics. 2012;32(2):453-468.

5. Gentzler RD, 2nd, Gault JH, Liedtke AJ, McCann WD, Mann RH, Hunter AS. Congenital absence of the left circumflex coronary artery in the systolic click syndrome. Circulation. 1975;52(3):490-496.

6. Tzeng YH, Lan GY, Yin WH, Huang CC, Wu YC. Cardiac computed tomography of congenital absent left circumflex coronary artery: a case report. Chinese Journal of Radiology. 2009;34(4):273-276.

7. Majid Y, Warade M, Sinha J, Kalyanpur A, Gupta T. Superdominant right sdcoronary artery with absent left circumflex artery. Biomed Imaging Interv J. 2011;7(1):e2.

8. Guo J, Xu M. Congenital absence of the left circumflex artery associated with inferior myocardial infarction. Intern Med. 2012;51(1):71-74.

9. McConnell SE, Collins KA. Sudden unexpected death resulting from an anomalous hypoplastic left coronary artery. J Forensic Sci. 1998;43(3):708-711.

10. Ali M, Hanley A, McFadden EP, Vaughan CJ. Coronary artery anomalies: a practical approach to diagnosis and management. Heart Asia. 2011;3(1):8-12.

11. Ali FS, Khan SA, Tai JM, Fatimi SH, Dhakam SH. Congenital absence of left circumflex artery with a dominant right coronary artery. BMJ Case Rep. 2009;2009.

12. Page HL, Jr., Engel HJ, Campbell WB, Thomas CS, Jr. Anomalous origin of the left circumflex coronary artery. Recognition, antiographic demonstration and clinical significance. Circulation. 1974;50(4):768-773. 